\title{
A Modeling Approach for Burn Scar Assessment Using Natural Features and Elastic Property
}

\author{
Yong Zhang, Student Member, IEEE, Dmitry B. Goldgof, Member, IEEE, Sudeep Sarkar, Member, IEEE, \\ and Leonid V. Tsap,
}

\begin{abstract}
A modeling approach is presented for quantitative burn scar assessment. Emphases are given to: (1) constructing a finite element model from natural image features with an adaptive mesh, and (2) quantifying the Young's modulus of scars using the finite element model and regularization method. A set of natural point features is extracted from the images of burn patients. A Delaunay triangle mesh is then generated that adapts to the point features. A 3D finite element model is built on top of the mesh with the aid of range images providing the depth information. The Young's modulus of scars is quantified with a simplified regularization functional, assuming that the knowledge of scar's geometry is available. The consistency between the Relative Elasticity Index and the physician's rating based on the Vancouver Scale (a relative scale used to rate burn scars) indicates that the proposed modeling approach has high potentials for image-based quantitative burn scar assessment.
\end{abstract}

Index Terms-Physical model, elastic property, burn scar, natural feature, finite element, regularization.

\section{INTRODUCTION}

$\mathbf{E}$ $\mathrm{ACH}$ year more than one million people suffer burn injuries in Canada and the US [14]. Accurate rating of scar condition is needed in order to design an effective treatment plan. Scar rating in clinical settings is done using the Vancouver rating scale or its variants by which experts assess the vascularity, pigmentation, pliability and size of scars [16], [22]. These rating methods suffer from their subjective nature and low consistency among rates. There is a strong need for a quantitative and objective scar rating method based on the biophysical properties of skin tissue [13].

In our previous works [20], [21], [23], we developed a comprehensive scar assessment method that utilizes the color, texture, pliability, thickness and size of scars. In this paper, we advance a physical model-based scar rating method which focuses on estimating the relative elasticity of scars. The contributions of the proposed method are: (1) noninvasive natural image features are used to generate an adaptive mesh and to build a finite element model. In previous works, we had used artificial markers (ink stamps) on the skin to facilitate

Manuscript received XXXXX, 2003; revised XXXXX, 2004. This work was partially supported by the Biomedical Engineering Research Grant from the Whitaker Foundation and the Grants EIA-0130768 from the NSF. This work was performed under the auspices of the U.S. Department of Energy by University of California Lawrence Livermore National Laboratory under contract number W-7405-Eng-48, UCRL-JRNL-203446.

Y. Zhang, D. Goldgof and S. Sarkar are with the Department of Computer Science and Engineering, University of South Florida. e-mail: [zhang,goldgof,sarkar]@csee.usf.edu.

L. Tsap is with the Electronics Engineering Department, University of California Lawrence Livermore National Laboratory. email: tsap1 @1lnl.gov. model construction. The use of natural features enables us to quantify the elastic property without the need of any markers, and hence greatly enhances the applicability of the proposed rating method; (2) a robust procedure of quantifying the scar elasticity is established using the regularization method so that the noisy data can be handled properly.

Although effort has been made to measure the scar elasticity directly using contact devices [1], there is need for noninvasive methods that infer scar elasticity from the observed tissue motion. Both ultrasonic and MR images have been used in tissue property reconstruction. Ultrasonic wave can penetrate soft tissues and is suitable for the study of internal organs [11], [12], [18]. But ultrasonic images are plagued by the noise artifacts and low resolution. MRI elastography has the advantage that motion can be measured with high resolution [10]. Creswell et al [3] used the MRI tagging technique and iterative finite element method for heart model evaluation. But MR imaging is expensive and less flexible. We will use regular optical and range images to quantify scar elasticity.

Two important issues in scar elasticity estimation are the ill-posedness and the computational cost. We experimented with the following approaches in the context of burn scar assessment: (1) reduce the parameter space by posing stronger constraints; (2) reduce the parameter space by using an adaptive mesh.

\section{MODEL CONSTRUCTION}

\section{A. Governing Equations}

The mechanical behavior of skin is determined by the presence of collagen fibers, elastin fibers and lubricating ground substance [6], [9], [19]. Burn scars tend to have random organization of collagen fibers during tissue regeneration. We assume that the mechanical behavior of burn scars can be approximated by an elastic and isotropic model. Let $\mathbf{u}$ be the displacement vector, $[e]=\left[e_{i j}, \gamma_{i j}\right]$ be the strain tensor, $[\sigma]=\left[\sigma_{i j}, \tau_{i j}\right]$ be the stress tensor, $\rho_{0}$ be the mass density, $\mathbf{B}$ be the body force, and $E$ and $\nu$ be the Young's modulus and the Poisson's ratio, the governing equation of elastic body motion can be obtained from three basic equations: (1) the strain compatibility equation, (2) the motion equation, and (3) the constitutive equation.

The strain compatibility equation ensures that the strain components give a single-valued continuous displacement:

$$
\nabla \times(\nabla \times e)^{T}=0
$$

The principle of conservation states that the rate of change of the total linear momentum of a continuous medium equals 
the sum of all the external forces:

$$
\rho_{0} \frac{\partial^{2} \mathbf{u}}{\partial t^{2}}=\nabla \cdot \sigma+\mathbf{B}
$$

The constitutive equation for linear and isotropic body is:

$$
\left[\begin{array}{c}
\sigma_{x x} \\
\sigma_{y y} \\
\sigma_{z z} \\
\tau_{x y} \\
\tau_{y z} \\
\tau_{z y}
\end{array}\right]=\frac{E}{(1+\nu)(1-2 \nu)} C\left[\begin{array}{c}
e_{x x} \\
e_{y y} \\
e_{z z} \\
\gamma_{x y} \\
\gamma_{y z} \\
\gamma_{z y}
\end{array}\right],
$$

where $C$ is the elastic coefficient tensor.

Combining equations (1), (2), and (3), we have the governing equation for elastic deformation:

$$
\rho_{0} \frac{\partial^{2} \mathbf{u}}{\partial t^{2}}=\nabla \cdot\left[\lambda(\nabla \cdot \mathbf{u}) \mathbf{I}+G \nabla \mathbf{u}+G(\nabla \mathbf{u})^{T}\right]+\mathbf{B},
$$

where $G$ and $\lambda$ are the Lamé constants, which can be computed from $E$ and $\nu$ :

$$
G=\frac{E}{2(1+\nu)}, \lambda=\frac{\nu E}{(1+\nu)(1-2 \nu)} .
$$

\section{B. Extraction of Natural Point Features}

We use the Shi-Tomasi detector to extract salient points in scar images [15], [17]. For each pixel $p$ in the image, we compute a $2 \times 2$ matrix $(g)$ within a window $(w)$ :

$$
g=\left[\begin{array}{ll}
\sum^{w} \frac{\partial I}{\partial x} \frac{\partial I}{\partial x} & \sum^{w} \frac{\partial I}{\partial x} \frac{\partial I}{\partial y} \\
\sum^{w} \frac{\partial I}{\partial y} \frac{\partial I}{\partial x} & \sum^{w} \frac{\partial I}{\partial y} \frac{\partial I}{\partial y}
\end{array}\right]
$$

where $I$ is the image intensity and $(x, y)$ represent row and column. We compute the first derivatives by convolving the intensity with the derivative of Gaussian filter $(G): \frac{\partial I}{\partial x}=$ $\frac{\partial G}{\partial x} * I, \quad \frac{\partial I}{\partial y}=\frac{\partial G}{\partial y} * I$. We then compute the coefficients of gradient matrix: $\frac{\partial I}{\partial x} \frac{\partial I}{\partial x}, \frac{\partial I}{\partial y} \frac{\partial I}{\partial y}, \frac{\partial I}{\partial x} \frac{\partial I}{\partial y}, \frac{\partial I}{\partial y} \frac{\partial I}{\partial x}$. We sum up the coefficients of all pixels inside $w$ to produce the matrix $g$ of pixel $p$. We then compute the eigenvalues of matrix $g$ : $(\lambda 1, \lambda 2)$. Given a threshold $T$, satisfaction of the condition: $\min (\lambda 1, \lambda 2)>T$, indicates that the window contains a corner/point with two strong edges along the eigenvector directions. We used a value of $T=1$ for all of the scar experiments. With this value, 400-800 features can be ensured in the scar images, which suffices the need of building a relatively dense finite element mesh.

We use another threshold $h$, the minimum distance between two adjacent points, to control feature distribution. Since scars have lower intensity than normal skins, we change the value of $h$ adaptively based on the intensity variation. We first select an area that contains only scars and skins. We then equalize the selected area to highlight the intensity contrast between scars and skins. For each feature point, its $h$ is computed based on the average intensity in $w$, scaled linearly by the overall intensity variation in the modeling area: $h=H_{\min }+\left(I_{w}-\right.$ $\left.I_{\min }\right) \frac{H_{\max }-H_{\min }}{I_{\max }-I_{\min }}$, where $h$ is the minimum distance between a feature and its neighbors, $I_{w}$ is the average intensity in $w$, $\left(I_{\max }, I_{\min }\right)$ are the maximum and minimum intensities in modeling area after equalization, and $\left(H_{\max }, H_{\min }\right)$ are userspecified maximum and minimum $h$ that correspond to $\left(I_{\max }\right.$,

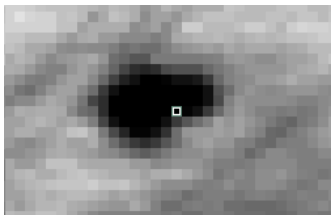

(a) Feature in frame 1

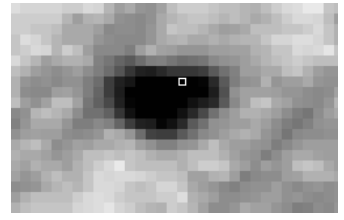

(b) Corresp. in frame 2
Fig. 1. Position shift between the corresponding features in two frames. Note that features in (a) and (b) are corresponding pairs. The white square indicates the computed feature position by the feature detector. The shift between (a) and (b) is 3 pixels.

TABLE I

THE PERFORMANCE OF POINT FEATURE DETECTOR WITH SCAR IMAGES

\begin{tabular}{|l|c|c|c|}
\hline & Min. & Max. & Average \\
\hline feature position shift (pixel) & 0 & 4.5 & 2.1 \\
actual displacement (pixel) & 11.9 & 36.2 & 24.8 \\
error (shift/displacement) & $0.0 \%$ & $13.3 \%$ & $7.2 \%$ \\
\hline
\end{tabular}

$\left.I_{\text {min }}\right)$. After computing $h$ for each feature, we sort all features based on the number of their neighbors that are in conflict with their $h$ value. By "conflict" we mean that the distance between a feature and its neighbors is less than its $h$. We then iteratively remove the feature that has the most conflicts until no conflict exists. The final distribution has more features in scars than in skins.

All images have the resolution of $640 \times 480$ pixels. Features are extracted using a window size of $9 \times 9$. The minimum distance $\left(H_{\min }\right)$ is in the range of $15-20$ pixels, and $H_{\max }$ is set to be $1.3-2.5$ times larger than $H_{\min }$. The choice of $H_{\min }$ depends on the size of scars. If $H_{\min }$ is too large, there may not be enough features in scar areas and vise versa. The same argument applies to the choice of $H_{\max }$. Those heuristic parameters must be tuned for a specific setting depending upon the quality of images and size of scars.

To quantify the precision of feature extraction, we examined 218 corresponding features in scar images. For each patient, two frames were taken (before and after deformation). Features were then extracted from two frames. There exist small shifts between the computed positions of corresponding features in two frames. An example is shown in Figure 1. The white square indicates the computed position by the feature detector. The correspondence pair has a shift of 3 pixels. Note that the feature patterns changed slightly between two frames due to the variations in lighting condition, projection and skin deformation. The actual displacements due to skin deformation between two correspondences are 26 pixels. Since we are interested in obtaining good displacement data for scar modeling, we define the extraction error as the ratio of the computed position shift to the actual displacement. For the example shown in Figure 1, the extraction errors is 11.5\%. For 218 feature pairs we examined, the results are summarized in Table I. With current imaging setting and feature extraction method, the average error introduced in feature extraction is less than $10 \%$. 


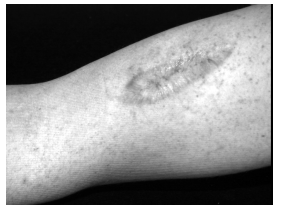

(a)

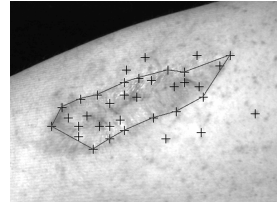

(b)

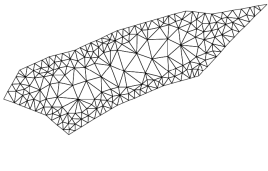

(c)
Fig. 2. Adaptive meshing using point features. (a) Scar image. (b) Point features and ROI. (c) Adaptive triangle mesh with refinement at the boundary.

\section{Adaptive Meshing}

Given a set of points distributed on object's surface, we can generate an adaptive mesh based on the Delaunay principle [2]. We use the following meshing procedure: (1) extract point features from images; (2) select the points inside the region of interest (ROI) for which a physical model will be built; (3) link the points on the boundary of ROI to form a closed polygon or surface; (4) generate an adaptive Delaunay mesh using feature points as its nodes; (5) refine the mesh.

Refinement is performed to insert new nodes into the area of interest as a quality assurance procedure. We follow these guidelines in the node insertion: (1) new nodes are added at the region of high curvature to ensure accurate surface representation; (2) new nodes should subdivide the thin element into regular elements to reduce modeling errors; (3) new nodes are added at regions of property discontinuities which could cause modeling errors. Figure 2 shows an example of adaptive triangle mesh.

\section{ESTIMATE SCAR ELASTICITY}

\section{A. Range Scanner Setting}

A K2T range scanner is used to obtain 3D displacement between corresponding features. The setting of K2T scanner is illustrated in Figure 3 (a). The K2T system consists of a CCD camera and a structured light projector, and computes the depth from images of striped light patterns. An example of structured light patterns is shown in Figure 3 (b). In scar study, the effective imaging area is about $30-35 \mathrm{~cm}$ cube. The distance between patients and the range camera is about 100$130 \mathrm{~cm}$.

A set of images (2-4 frames) was taken while patient's skin was stretched. Since the scar condition of most patients did not allow the use of contact devices, patient's skin was pulled gently by hands to avoid pain and further damage. Therefore, it was not possible to measure the forces applied to patient's skin. 3D displacement $(\mathbf{u})$ obtained from intensity and range images is used to compute the objective function in an "outputleast-squared" form: $\|F(E)-\mathbf{u}\|^{2}$, where $F(E)$ denotes the forward model.

\section{B. Regularization and Assumptions}

We use a Poisson's ratio of 0.495 by approximating the skin tissue as near-incompressible material. We consider the governing equation of static case:

$$
\nabla \cdot\left[\lambda(\nabla \cdot \mathbf{u}) \mathbf{I}+G \nabla \mathbf{u}+G(\nabla \mathbf{u})^{T}\right]+\mathbf{B}=0,
$$

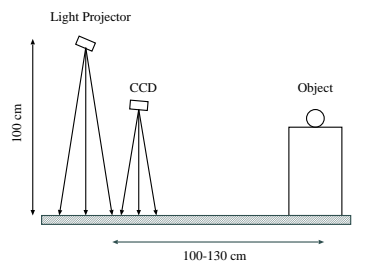

(a) Imaging geometry

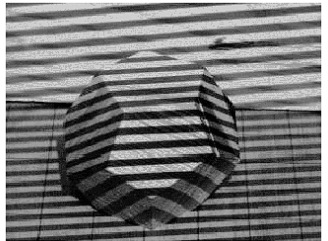

(b) Light stripe pattern
Fig. 3. The geometry of K2T range scanner for burn scar study.

and define a differential operator $A(E)$ as:

$$
A(E)=\nabla \cdot\left[\lambda(\nabla \cdot(\cdot)) \mathbf{I}+G \nabla(\cdot)+G(\nabla(\cdot))^{T}\right] .
$$

Using $A(E)$, we obtain a nonlinear operator equation:

$$
F(E)=-\mathbf{B} A(E)^{-1}=\mathbf{u} .
$$

Because of the discontinuous dependence of solution $(E)$ on noisy data $(\mathbf{u})$, the solution obtained by minimizing the "output-least-squared" objective function is numerically unstable and certain forms of regularization must be imposed [5]. We use the Tikhonov functional in its variational form:

$$
T(E)=\|F(E)-\mathbf{u}\|^{2}+\alpha\left\|W\left(E-E^{*}\right)\right\|^{2},
$$

where $E^{*}$ denotes the prior knowledge of the inverse solution, $\alpha$ is the regularization parameter and $W$ is the smoothness matrix. The gradient methods can be used to find the minimizer of the Tikhonov functional, provided that they start with a good initial guess. Applications of the Tikhonov functional to elasticity reconstruction of soft tissues have been reported in [4], [8].

We are interested in determining the relative elasticity of scars. To reduce the computational complexity, we make several assumptions: (1) the geometry of scars is known; (2) the elasticity of scar is higher than that of normal skin; (3) the elasticity of background normal skin is known. We then transform the minimization of (10) into a one-dimensional search problem with two steps:

1) Determine the regularization parameter using the Lcurve method [7].

2) Change the elasticity of scars $\left(E_{s}\right)$ until the minimizer of the simplified functional is found:

$$
T\left(E_{s}\right)=\left\|F\left(E_{s}\right)-\mathbf{u}\right\|^{2}+\alpha\left\|W\left(E_{s}-E_{s}^{*}\right)\right\|^{2} .
$$

In L-curve method, the $\log$ of the regularized solution norm $\log \|E\|$ is plotted against the $\log$ of the residual norm $\log \|F(E)-\mathbf{u}\|$ for a range of $\alpha$. The optimal $\alpha$ is chosen to be the one that corresponds to the corner of the L-curve.

\section{Boundary Condition Specification}

We specify displacements at the boundary nodes that are also feature points. We designate the feature points inside the modeling domain as the controlling nodes, on which the measured displacements and the simulated displacements by forward model are used to calculate the residual norm. Since 
TABLE II

THE ESTIMATED RELATIVE SCAR ELASTICITY (KPA)

\begin{tabular}{|c|c|c|c|c|}
\hline Patients & $\begin{array}{c}\text { Scar elasticity } \\
\text { (Natural features) }\end{array}$ & $\begin{array}{c}\text { Scar elasticity } \\
\text { (Artifi. markers) }\end{array}$ & $\begin{array}{c}\text { Absolute } \\
\text { error }\end{array}$ & $\begin{array}{c}\text { Relative } \\
\text { error }\end{array}$ \\
\hline 970407 & 53 & 46 & 7 & $13.2 \%$ \\
970416 & 12 & 14 & 2 & $16.6 \%$ \\
970425 & 41 & 37 & 4 & $9.7 \%$ \\
970922 & 19 & 18 & 1 & $5.3 \%$ \\
\hline
\end{tabular}

Errors are computed between the results using natural features and artificial markers. The elasticity of normal skin used in experiments is $5 \mathrm{kPa}$.

only displacement is available, we use the elasticity of normal skins as a reference to determine the relative elasticity of scars. The reported Young's modulus values of soft tissues are in the range of 1-100 $\mathrm{kPa}$ [6], [19]. In scar assessment, we used a Young's modulus of $5 \mathrm{kPa}$ for normal skin.

It is worth noting that the body force (B) in (7) and (9) should not be confused with the boundary conditions in normal sense such as surface force/traction. The variation of body force (mainly gravity) is a significant factor in the simulation of large-sized objects such as the sea water in a bay or the planet earth. But for small objects such as scars, body force can be treated as a constant.

\section{EXPERIMENTS}

\section{A. Data Set}

The data set includes images of four patients that were taken at different healing stages. The boundaries between scars and normal skins can be clearly identified in those images.

\section{B. Quantify Scar Elasticity}

We carried out two independent experiments, one using artificial markers and one using natural features, to determine if the natural feature is a viable option for replacing the artificial marker. The results for all patients are listed in Table II. Although direct measurements of scar's Young's modulus are not available for those patients, the fact that the differences between the estimated Young's modulus using artificial markers and using natural features are very small suggests a high level of consistency. The results of patient970922 are plotted in Figure 4. As expected, experiment using artificial markers shows a slightly better performance than that using natural features, as indicated by the minimization curves (Figure 4 (f)). But the disparity between two curves is very small. More importantly, the minimum points of two curves are almost identical $(18 \mathrm{kPa}$ and $19 \mathrm{kPa})$. The strain distributions also match well with scar distribution.

The errors in measured displacements mainly came from two sources: (1) feature extraction caused by illumination and texture variations; (2) correspondence mismatch. Since we manually established the correspondence, the disparity between the modeling results of using natural features and using artificial markers is mainly caused by the feature extraction error. As analyzed before, the feature extraction error is less than $10 \%$ on average, which is an acceptable value for burn scar assessment.

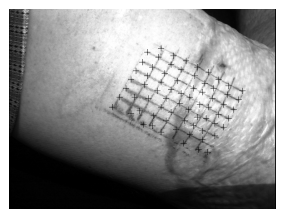

(a)

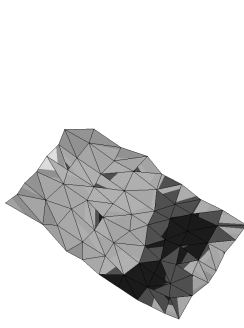

(d)

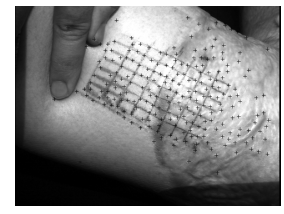

(b)

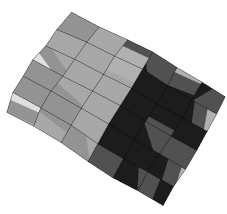

(e)

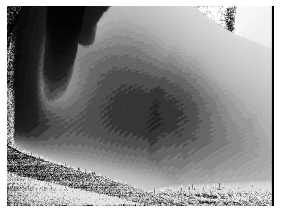

(c)

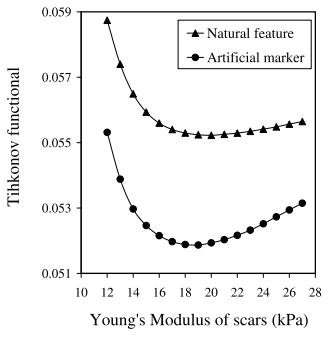

(f)
Fig. 4. Estimated elasticity and strain (patient-970922). (a) Scar image. (b) After being stretched. (c) Range image. (d) Strain using natural features. (e) Strain using markers. (f) Minimization curves. In (d) and (e), dark color indicates low strain value of scars.

\section{Relative Elastic Index}

Current clinical scar assessment methods are based on a relative rating scale. We want to investigate if there exists a positive correlation between the clinical rating and the estimated elasticity ratio of scars to normal skins, so that we can establish a standard that is quantitative and comparable to the one used by physicians. We define the Relative Elastic Index (REI) as the ratio of average Young's modulus of scars to that of normal skins:

$$
R E I=\frac{\sum_{i=1}^{n} E_{i} / n}{\sum_{j=1}^{m} E_{j} / m}
$$

where $n$ and $m$ are the numbers of elements inside scars and normal skins, respectively.

In Table III, we list physician's ratings, as well as REIs using both artificial markers and natural features. In all experiments, patient's skin was stretched along two directions (horizontal and vertical). So, each patient was studied twice. In Figure 5, the REIs using natural features and artificial markers are plotted against the physician's ratings. Two important observations can be made: (1) there exists a good correlation between the physician's ratings and the REIs using either natural features or artificial markers; (2) the REIs computed using natural feature are consistent with those using artificial markers, especially for less damaged scars. The slight deviation from the linear monotonic relationship is probably caused by the complex scar patterns, which affect both feature extraction and matching. This problem can be solved by calibrating the model against the ground truth, a possibility that is currently under investigation. It should be pointed out that, due to the limited number of patients, we do not draw any statistical conclusion at this point about the correlation between the physicians' rating and the REI. 
TABLE III

PHySiCIAN'S RATING AND RELATIVE ElASTIC INDEX (REI)

\begin{tabular}{|c|c|c|c|}
\hline & $\begin{array}{c}\text { Physician's } \\
\text { rating }\end{array}$ & $\begin{array}{c}\text { REI using } \\
\text { natural features }\end{array}$ & $\begin{array}{c}\text { REI using } \\
\text { artificial markers }\end{array}$ \\
\hline scar-970407(hori) & 4.5 & 9.5 & 8.0 \\
scar-970407(vert) & 4.5 & 9.1 & 7.3 \\
scar-970416(hori) & 3.0 & 2.8 & 3.3 \\
scar-970416(vert) & 3.0 & 4.1 & 3.8 \\
scar-970425(hori) & 3.3 & 6.3 & 5.0 \\
scar-970425(vert) & 3.3 & 7.4 & 5.6 \\
scar-970922(hori) & 2.0 & 2.2 & 2.3 \\
scar-970922(vert) & 2.0 & 2.8 & 2.0 \\
\hline normal-skin(hori) & 1.0 & 1.0 & 1.0 \\
normal-skin(vert) & 1.0 & 1.0 & 1.0 \\
\hline
\end{tabular}

For each patient, the skin was stretched in two directions, horizontally and vertically. The REI of normal skin (1.0) is defined as the baseline.

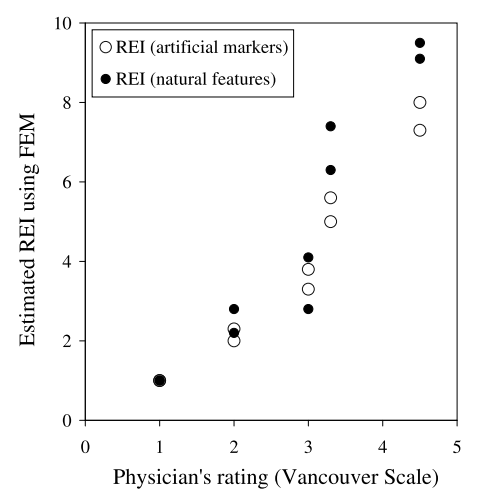

Fig. 5. Correlation between physician's rating and REIs using natural features and artificial markers.

\section{CONCLUSIONS}

We present a modeling approach for quantitative burn scar assessment. We construct a finite element model using a Delaunay mesh that is adapted to natural image features. The use of natural features and adaptive mesh not only increases the computational efficiency, but also allows us to work with scar images without the need of tagging artificial markers. We find that the difference between the modeling results of using artificial markers and using natural features is negligible, indicating that natural feature is a viable option for burn scar assessment. We quantify burn scar damage by estimating its Young's modulus using a simplified regularization method. Experiments show that the proposed method is robust when presented with noisy data. The positive correlation between the physician's rating and REIs (using both natural features and artificial markers) suggests that the proposed approach can provide a quantitative and objective evaluation of burn scar damage.

It should be stressed that, although the linear model is widely used in physics-based simulations, the biomechanical behavior of soft tissues is intrinsically nonlinear. We choose the linear model for this study based on the assumption that the deformation of scar/skin is relatively small so that it can be approximated by a linear model. In future investigations, we will revisit the nonlinear aspects of burn scar modeling, both geometrically (Green strain tensor) and materially (viscoelastic and plastic materials).

\section{REFERENCES}

[1] T. H. Bartell, W. W. Monafo, and T. A. Mustoe, "A new instrument for serial measurement of elasticity in hypertropic scar," Journal of Burn Care and Rehabilitation, vol. 9, pp. 657-660, 1988.

[2] G. F. Carey, Computational Grids : Generation, Adaptation, and Solution Strategies. Series in Computational and Physical Processes in Mechanics and Thermal Science. Taylor \& Francis, 1997.

[3] L. L. Creswell, M. J. Moulton, S. G. Wyers, J. S. Pirolo, D. S. Fishman, W. H. Perman, K. W. Myers, R. L. Actis, M. W. Vannier, B. A. Szabo, and M. K. Pasque, "An experimental method for evaluating constitutive models of myocardium in in vivo hearts," American J. of Physiology, Heart and Circulatory Physiology, vol. 267, pp. 853-863, 1994.

[4] M. M. Doyley, P. M. Meaney, and J. C. Bamber, "Evaluation of an iterative reconstruction method for quantitative elastography," IEEE Phys. Med. Biol., vol. 45, pp. 1521-1540, 2000.

[5] H. W. Engl, M. Hanke, and A. Neubauer, Regularization of Inverse Problems, Kluwer Academic Publishers, c1996.

[6] Y. C. Fung, Biomechanics; Mechanical Properties of Living Tissues, Springer-Verlag, 2nd ed. 1993.

[7] P. C. Hansen, "Analysis of discrete ill-posed problems by means of the L-curve," SIAM Rev., vol. 34, pp. 561-580, 1992.

[8] F. Kallel and M. Bertrand, "Tissue elasticity reconstruction using linear perturbation method," IEEE Trans. Med. Imag., vol. 15, no. 3, pp. 299313, 1996.

[9] W. F. Larrabee and J. A. Galt, "A finite element model of skin deformation. (iii) the finite element model," Laryngoscope , vol. 96, pp. 413-419, 1986.

[10] R. Muthupillai, D. J. Lomas, P. J. Rossman, J. F. Greenleaf, A Manduca, and R. L. Ehman, "Magnetic resonance elastography by direct visualization of propagating acoustic strain waves," Science, vol. 269, pp. 1854-1857, 1995.

[11] J. Ophir, I. Cespedes, H. Ponnekanti, Y. Yazdi, and X. Li, "Elastography: A quantitative method for measuring the elasticity of biological tissues," Ultrasonic Imaging, vol. 13, pp. 111-134, 1991.

[12] K. J. Parker, L. Gao, R. M. Lerner, and S. F. Levinson, "Techniques for elastic imaging: a review," IEEE Engineering in Medicine and Biology, vol. 15, pp. 52-59, 1996.

[13] P. S. Powers, S. Sarkar, D. B. Goldgof, C. W. Cruse, and L. V. Tsap, "Scar Assessment: Current problems and future solutions," Journal of Burn Care and Rehabilitation, vol. 20, no. 1, Jan. 1999.

[14] J. R. Saffle, B. Davis, and P. Williams, "Recent outcomes in the treatment of burn injury in the United States: A report from the American Burn Association Patient Registry," Journal of Burn Care and Rehabilitation, vol. 16, pp. 219-232, May 1995.

[15] C. Schmid, R. Mohr, and C. Bauckhage, "Evaluation of interest point detectors," International Journal of Computer Vision, vol. 37, no. 2, pp. $151-172,2000$

[16] T. Sullivan, J. Smith, J. Kermode, E. McIver, and D. J. Courtemanche, "Rating the burn scar," Journal of Burn Care Rehabilatation, vol. 11, pp. 256-260, 1990.

[17] J. Shi and C. Tomasi, "Good features to track," IEEE Conf. on Computer Vision and Pattern Recognition, Seattle, pp. 593-600, June 1994.

[18] A. R. Skovoroda, S. Y. Emelianov, M. A. Lubinski, A. P. Sarvazyan, and M. O'Donnell, "Theoretical analysis and verification of ultrasound displacement and strain imaging," IEEE Trans. Ultrasonics, Ferroelectrics, and Frequency Control, vol. 41, no. 3, pp. 302-313, 1994.

[19] J. G. Thacker, "The elastic properties of human skin in vivo," Ph.D. Dissertation, University of Virginia, Charlottesville, VA, 1976.

[20] L. V. Tsap, D. B. Goldgof, S. Sarkar, and P. S. Powers, "A visionbased technique for objective assessment of burn scars," IEEE Trans. Med. Imag., vol. 17, no. 4, pp. 620-633, 1998.

[21] L. V. Tsap, D. B. Goldgof, and S. Sarkar, "Nonrigid motion analysis based on dynamic refinement of finite element models," IEEE Trans. Pattern Analysis and Machine Intell., vol. 22, no. 5, pp. 526-543, 2000.

[22] E. K. Yeong, R. Mann, L. H. Engrav, M. Goldberg, V. Cain, B. Costa, M. Moore, D. Nakamura, J. Lee, "Improved burn scar assessment with use of a new scar-rating scale," Journal of Burn Care and Rehabilitation, vol. 18 , no. 4, pp. 353-355, 1997.

[23] Y. Zhang, D. B. Goldgof, S. Sarkar, and L. V. Tsap, "Model-based nonrigid motion analysis using natural feature adaptive mesh," Proceedings of International Conference on Pattern Recognition, pp. 839-843, 2000. 